\title{
Implications of Changes in Medicare Payment and Documentation for Primary Care Spending and Time Use
}

J Gen Intern Med 36(3):836-9

DOI: $10.1007 / \mathrm{s} 11606-020-05857-4$

(C) Society of General Internal Medicine 2020

\section{INTRODUCTION}

Primary care is - ideally — accessible, timely, first-contact care that is comprehensive, coordinated, and longitudinal. To support primary care practices, several states have passed regulations or legislation to raise primary care spending to $7-12 \%$ of total healthcare spending. ${ }^{1,2}$ The Centers for Medicare and Medicaid Services (CMS) has finalized the first major change to primary care payment in the Physician Fee Schedule in decades - raising fees for evaluation and management visits, and lowering documentation requirements (obviating rulebased review of systems and exam elements in favor of time-based or decision-complexity-based coding), beginning $20211^{3}$

We sought to estimate the extent to which the new CMS rule may reach target primary care spending levels pursued in several states, ${ }^{1,2}$ and the degree to which reduced documentation requirements may affect time use in primary care practices.

\section{METHODS}

We used a previously-validated model, ${ }^{4}$ which simulates the national population of primary care providers and their patients, reflecting current patterns of utilization, spending, and practice-level revenues and costs from all payers. Primary care spending was defined as the sum of all payments to practitioners in general practice, general internal medicine, or family practice, and included geography-specific Medicare payments by visit code (data from $N=181,664$ primary care providers, Table 1). ${ }^{5}$ Reduced documentation time was estimated based on a prior practice time use study, ${ }^{6}$ and disaggregated into time spent during visits, outside of visits during practice hours, and after practice hours (Table 1). To estimate potential all-payer effects, we re-calculated the outcomes if commercial insurers and Medicaid adopted the same proportionate rate increase and documentation rules as Medicare. We also identified the implications of potential up-coding of visits to higher levels of complexity given the reduced documentation requirements.

\section{RESULTS}

Medicare Only. Based solely on the fee increases as applied to Medicare patients, we anticipate the CMS policy to increase gross revenues to primary care practices by $\$ 20,176 /$ full-time physician/year (95\% CI $\$ 1766$ to $\$ 90,261)$-equivalent to an increase of 0.3 percentage points nationwide from the current average of $2.1 \%$ of total Medicare spending devoted to primary care. The increase would not be expected to achieve state targets for primary care spending (Table 2).

One-third of the usual time per practitioner note $(\sim 1.2 \mathrm{~min} /$ visit note) was estimated to be eliminated by the change in documentation requirements. Time saved from documentation would be expected to be $14.9 \mathrm{~min} /$ practitioner/day (95\% CI 2.7 to 27.4 ), with $9.5 \mathrm{~min} /$ day saved from documentation during visits, $3.7 \mathrm{~min}$ outside of visits during practice hours, and 1.7 min outside of practice hours. 
Table 1 Model Input Data and Sources

\begin{tabular}{|c|c|c|}
\hline Parameter & Value & Source \\
\hline \multicolumn{3}{|l|}{ Medicare payments } \\
\hline \multicolumn{3}{|l|}{2019 Schedule estimate (reference) by CPT code* } \\
\hline 99,201 & $\$ 46.49$ & Centers for Medicare and Medicaid Services ${ }^{3}$ (based on \\
\hline 99,202 & $\$ 77.12$ & relative value units listed in Table 125 of referenced rule) \\
\hline 99,203 & $\$ 109.20$ & \\
\hline 99,204 & $\$ 166.86$ & \\
\hline 99,205 & $\$ 210.83$ & \\
\hline 99,211 & $\$ 23.43$ & \\
\hline 99,212 & $\$ 46.13$ & \\
\hline 99,213 & $\$ 76.04$ & \\
\hline 99,214 & $\$ 110.28$ & \\
\hline 99,215 & $\$ 148.12$ & \\
\hline \multicolumn{3}{|c|}{$\begin{array}{l}\text { code modifier (GPC1X) } \\
\text { coded Schedule estimate (updated rule) by CPT code*, including new primary care G }\end{array}$} \\
\hline 99,201 & $\$ 0$ (to be discontinued) & \\
\hline 99,202 & $\$ 89.38$ & \\
\hline 99,203 & $\$ 128.30$ & \\
\hline 99,204 & $\$ 184.88$ & \\
\hline 99,205 & $\$ 233.53$ & \\
\hline 99,211 & $\$ 34.96$ & \\
\hline 99,212 & $\$ 60.55$ & \\
\hline 99,213 & $\$ 99.11$ & \\
\hline 99,214 & $\$ 133.34$ & \\
\hline 99,215 & $\$ 184.52$ & \\
\hline $\begin{array}{l}\text { Medicare patients as a proportion of primary care } \\
\text { patients, at practices accepting Medicare }\end{array}$ & $31.3 \%(95 \%$ CI $4.9 \%, 66.7 \%)$ & National Ambulatory Medical Care Survey, details in ${ }^{4}$ \\
\hline $\begin{array}{l}\text { Medicare patients as a proportion of primary care } \\
\text { visits }\end{array}$ & $24.7 \%$ (SD: 1.1$)$ & National Ambulatory Medical Care Survey, details in ${ }^{4}$ \\
\hline Documentation time (hours/visit/day) & & Prior time study ${ }^{6}$ \\
\hline During-visit & 1.33 (SD: 0.86) & \\
\hline Outside of visit, during practice hours & 0.52 (SD: 0.72 ) & \\
\hline Outside of practice hours & 0.24 (SD: 0.73$)$ & \\
\hline Visits/provider/day & $12.3(\mathrm{SD}: 5.3)$ & \\
\hline $\begin{array}{l}\text { Current fee-for-service payments to primary care, } \\
\text { per full-time provider per year (all payers) }\end{array}$ & $\begin{array}{l}\$ 638,634 \text { (SD: } \$ 481,774 ; \text { IQR: } \\
\$ 378,463, \$ 765092)\end{array}$ & Medical Group Management Association, details in ${ }^{4}$ \\
\hline
\end{tabular}

*Non-facility national average estimates are provided. Area-specific variations including work, practice expense, and malpractice geographic practice cost indices were taken accounted for in state-specific estimates (see Table 2). CPT: Current Procedural Terminology; 95\% CI: 95\% confidence intervals; SD: standard deviation; IQR: interquartile range

Additional Payers. If commercial insurers and Medicaid were to adopt the Medicare policy, the overall composite fee increases would be anticipated to increase gross revenues by $\$ 156,071 /$ full-time physician/year $(95 \% \mathrm{CI}$ $\$ 62,028$ to $\$ 239,738$ ) - an increase of 1.2 percentage points in the share of total national health care spending allocated to primary care, from the current share of $5.6 \%$ across all payers (including Medicare). This spending would approach state targets (Table 2). Time saved from documentation (including Medicare, Medicaid, and commercially insured patients) would be expected to be $41.8 \mathrm{~min} /$ practitioner/day (95\% CI 39.4 to 44.2 ), with $26.6 \mathrm{~min} /$ day saved from documentation during visits, 10.4 min outside of visits during practice hours, and 4.8 min outside of practice hours.
Incorporating Potential Up-Coding Effects. If 5\% of visits previously coded as level 3 or level 4 were up-coded by one level (to levels 4 and 5, respectively), we would expect the Medicare proportion of spending on primary care to increase by 0.4 percentage points, and-if other payers followed Medicare - total spending on primary care to increase by 1.5 percentage points.

\section{DISCUSSION}

Medicare payment changes that will take effect in 2021 will likely be insufficient to reach state target levels of primary care spending alone, but if all other payers follow Medicare, primary care spending levels may approach target levels. 
Table 2 Results from Simulation of Medicare Payment Increase

\begin{tabular}{|c|c|c|c|c|c|}
\hline & $\begin{array}{l}\text { Current Medicare } \\
\text { primary care } \\
\text { spending as } \% \text { of } \\
\text { total Medicare } \\
\text { spending }\end{array}$ & $\begin{array}{l}\text { Future Medicare } \\
\text { primary care } \\
\text { spending as \% of total } \\
\text { Medicare spending } \\
\text { (model estimates) }\end{array}$ & $\begin{array}{l}\text { Current all-payer } \\
\text { primary care spend- } \\
\text { ing as \% of total } \\
\text { healthcare spending }\end{array}$ & $\begin{array}{l}\text { Future all-payer pri- } \\
\text { mary care spending as } \\
\% \text { of total healthcare } \\
\text { spending (model esti- } \\
\text { mates)* }\end{array}$ & $\begin{array}{l}\text { Target primary care } \\
\text { spending, as \% of total } \\
\text { healthcare spending (if } \\
\text { legislation/regulation } \\
\text { passed) }{ }^{\dagger}\end{array}$ \\
\hline $\begin{array}{l}\text { National } \\
\text { average }\end{array}$ & $2.1 \%$ & $2.4 \%$ & $5.6 \%$ & $6.8 \%$ & - \\
\hline Alabama & $2.3 \%$ & $2.6 \%$ & $6.2 \%$ & $7.4 \%$ & - \\
\hline Alaska & $2.0 \%$ & $2.3 \%$ & \pm & $t$ & - \\
\hline Arizona & $2.5 \%$ & $2.8 \%$ & $5.2 \%$ & $6.4 \%$ & - \\
\hline Arkansas & $2.1 \%$ & $2.4 \%$ & $\$$ & + & - \\
\hline California & $2.2 \%$ & $2.5 \%$ & $6.1 \%$ & $7.3 \%$ & Pending \\
\hline Colorado & $2.2 \%$ & $2.5 \%$ & $5.0 \%$ & $6.2 \%$ & $7.2 \%$ (commercial) \\
\hline Connecticut & $1.9 \%$ & $2.2 \%$ & $3.5 \%$ & $4.7 \%$ & - \\
\hline Delaware & $2.3 \%$ & $2.6 \%$ & \pm & $t$ & Pending \\
\hline \multicolumn{6}{|l|}{ Columbia } \\
\hline Florida & $2.3 \%$ & $2.6 \%$ & $5.7 \%$ & $6.9 \%$ & - \\
\hline Georgia & $2.2 \%$ & $2.5 \%$ & $5.7 \%$ & $6.9 \%$ & - \\
\hline Hawaii & $3.2 \%$ & $3.5 \%$ & $\dagger$ & $t$ & Pending \\
\hline Idaho & $1.7 \%$ & $2.0 \%$ & 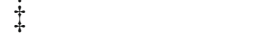 & $t$ & - \\
\hline Illinois & $2.1 \%$ & $2.4 \%$ & $5.0 \%$ & $6.2 \%$ & - \\
\hline Indiana & $1.9 \%$ & $2.2 \%$ & $4.7 \%$ & $5.9 \%$ & - \\
\hline Iowa & $2.1 \%$ & $2.4 \%$ & 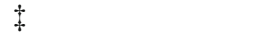 & $t$ & - \\
\hline Kansas & $1.9 \%$ & $2.2 \%$ & $\ddagger$ & $t$ & - \\
\hline Kentucky & $1.9 \%$ & $2.2 \%$ & $4.5 \%$ & $5.7 \%$ & - \\
\hline Louisiana & $1.6 \%$ & $1.9 \%$ & $5.3 \%$ & $6.5 \%$ & - \\
\hline Maine & $1.9 \%$ & $2.2 \%$ & $t$ & 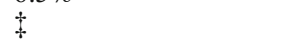 & Pending \\
\hline Maryland & $2.2 \%$ & $2.5 \%$ & $5.5 \%$ & $6.7 \%$ & - \\
\hline Massachusetts & $2.1 \%$ & $2.4 \%$ & $4.8 \%$ & $6.0 \%$ & Pending \\
\hline Michigan & $2.1 \%$ & $2.4 \%$ & $4.7 \%$ & $5.9 \%$ & - \\
\hline Minnesota & $1.9 \%$ & $2.2 \%$ & $7.6 \%$ & $8.8 \%$ & - \\
\hline Mississippi & $1.7 \%$ & $2.0 \%$ & $t$ & $\dagger$ & - \\
\hline Missouri & $1.8 \%$ & $2.1 \%$ & $4.6 \%$ & $5.8 \%$ & Pending \\
\hline Montana & $1.7 \%$ & $2.0 \%$ & $t$ & $t$ & - \\
\hline Nebraska & $1.8 \%$ & $2.1 \%$ & + & $\ddagger$ & - \\
\hline Nevada & $2.0 \%$ & $2.3 \%$ & $t$ & \pm & - \\
\hline New & $2.0 \%$ & $2.3 \%$ & $t$ & $t$ & - \\
\hline \multicolumn{6}{|l|}{ Hampshire } \\
\hline New Jersey & $2.1 \%$ & $2.4 \%$ & $4.6 \%$ & $5.8 \%$ & - \\
\hline New Mexico & $2.2 \%$ & $2.5 \%$ & 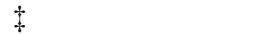 & \pm & - \\
\hline New York & $2.1 \%$ & $2.4 \%$ & $5.0 \%$ & $6.2 \%$ & - \\
\hline North Carolina & $2.4 \%$ & $2.7 \%$ & $5.9 \%$ & $7.1 \%$ & - \\
\hline North Dakota & $1.6 \%$ & $1.9 \%$ & 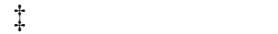 & \pm & - \\
\hline Ohio & $2.1 \%$ & $2.4 \%$ & $4.6 \%$ & $5.8 \%$ & - \\
\hline Oklahoma & $2.0 \%$ & $2.3 \%$ & $6.7 \%$ & $7.9 \%$ & - \\
\hline Oregon & $2.0 \%$ & $2.3 \%$ & $5.6 \%$ & $6.8 \%$ & $12.0 \%$ \\
\hline Pennsylvania & $2.2 \%$ & $2.5 \%$ & $4.2 \%$ & $5.4 \%$ & - \\
\hline Rhode Island & $2.3 \%$ & $2.6 \%$ & \pm & \pm & $10.7 \%$ (commercial) \\
\hline South Carolina & $2.4 \%$ & $2.7 \%$ & $5.0 \%$ & $6.2 \%$ & \\
\hline South Dakota & $1.6 \%$ & $1.9 \%$ & 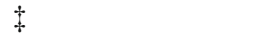 & $t$ & - \\
\hline Tennessee & $2.2 \%$ & $2.5 \%$ & $4.8 \%$ & $6.0 \%$ & - \\
\hline Texas & $1.9 \%$ & $2.2 \%$ & $6.3 \%$ & $7.5 \%$ & - \\
\hline Utah & $2.2 \%$ & $2.5 \%$ & + & + & - \\
\hline Vermont & $2.4 \%$ & $2.7 \%$ & 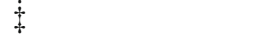 & $i$ & Pending \\
\hline Virginia & $2.6 \%$ & $2.9 \%$ & $5.7 \%$ & $6.9 \%$ & - \\
\hline Washington & $2.1 \%$ & $2.4 \%$ & $5.9 \%$ & $7.1 \%$ & - \\
\hline West Virginia & $2.2 \%$ & $2.5 \%$ & $t$ & \pm & Pending \\
\hline Wisconsin & $2.0 \%$ & $2.3 \%$ & $6.2 \%$ & $7.4 \%$ & - \\
\hline Wyoming & $1.7 \%$ & $2.0 \%$ & $\ddagger$ & $\ddagger$ & - \\
\hline \multicolumn{6}{|c|}{ International comparators } \\
\hline \multicolumn{3}{|c|}{$\begin{array}{l}\text { Organization for Economic Coc } \\
\text { (OECD), mean of } 23 \text { countries }\end{array}$} & $12.0 \%$ & - & - \\
\hline \multicolumn{3}{|c|}{ United Kingdom, National Health Service } & $8.4 \%$ & - & - \\
\hline \multicolumn{3}{|c|}{ Canada, Ontario province } & $8.1 \%$ & - & - \\
\hline
\end{tabular}

Estimates include adjustments for Geographic Practice Cost Indices, as well as work, practice expense, and malpractice relative value units. ${ }^{3}$ Current spending estimates and target spending levels from the Milbank Memorial Fund. ${ }^{1}$ International comparisons from Koller et al. ${ }^{2}$ The term "pending" in the last column indicates that legislation or regulation has passed state legislature, requiring the setting of a spending target, but that the department or committee charged with target setting has not yet agreed to or released a target publicly, to our knowledge

*If other payers (commercial, Medicaid) adopt the same proportionate rate increase and documentation rules as Medicare

†As of October 2019

IInsufficient data 
Sanjay Basu, $M D, P h D^{1,2,3}$

Zirui Song, $M D, P h D^{1,4,5}$

Russell S. Phillips, $M D^{1,6}$

Asaf Bitton, $M D, M^{1,4,7,8}$

Bruce E. Landon, $M D, M B A^{1,4,6}$

${ }^{1}$ Center for Primary Care, Harvard Medical School,

Boston, MA, USA

${ }^{2}$ Research and Analytics, Collective Health,

San Francisco, CA, USA

${ }^{3}$ School of Public Health, Imperial College London,

London, UK

${ }^{4}$ Department of Health Care Policy, Harvard Medical School,

Boston, MA, USA

${ }^{5}$ Department of Medicine, Massachusetts General Hospital,

Boston, MA, USA

${ }^{6}$ Division of General Medicine, Beth Israel Deaconess Medical Center,

Boston, MA, USA

${ }^{7}$ Division of General Medicine, Brigham and Women's Hospital,

Boston, MA, USA

${ }^{8}$ Ariadne Labs, Brigham and Women's Hospital and Harvard T.H. Chan School of Public Health, Boston, MA, USA

Corresponding Author: Sanjay Basu, MD, PhD; Center for Primary Care, Harvard Medical School Boston, MA, USA (e-mail: Sanjay_basu@hms.harvard.edu).

Data Availability Modeling was performed in $R$ with data and code for replication posted at: https://github.com/sanjaybasu/ medicarerateincrease

\section{Compliance with Ethical Standards:}

Conflict of Interest: Dr. Basu reports grants from the US National Institutes of Health; grants from the US Centers for Disease Control and Prevention; grants from the US Department of Agriculture; grants from the Robert Wood Johnson Foundation; personal fees from the Research Triangle Institute; personal fees from Collective Health, from HealthRight360, from KPMG; personal fees from PLOS Medicine; personal fees from The New England Journal of Medicine, outside the submitted work.

Dr. Bitton reports personal fees from the Centers for Medicare and Medicaid Innovation, outside the submitted work.

Dr. Phillips has nothing to disclose.

Dr. Song reports grants from the National Institutes of Health, Office of the Director, during the conduct of the study; personal fees from International Foundation of Employee Benefit Plans, outside the submitted work.

Dr. Landon reports non-financial support and other from Myers-JDC Brookdale Institute, other from City of Newton, MA, other from Health Resources in Action Board, personal fees from Research Triangle Institute International, personal fees from American Board of Internal Medicine (ABIM), personal fees from UpToDate, Inc., personal fees from Freedman Healthcare Consulting, grants from NIH, outside the submitted work.

\section{REFERENCES}

1. Primary Care Investment: State Policy and Spending Maps | Milbank Memorial Fund [Internet]. Milbank Memorial Fund. [cited 2019 Dec 9]. Available from: https://www.milbank.org/programs/primary-care-spend/ other-resources /

2. Koller CF, Khullar D. Primary Care Spending Rate - A Lever for Encouraging Investment in Primary Care [Internet]. N Engl J Med. 2017;377:1709-11. https://doi.org/10.1056/nejmp1709538

3. CMS-1715-F | CMS [Internet]. [cited 2019 Dec 2]. Available from: https:// www.cms.gov/Medicare/Medicare-Fee-for-Service-Payment/ PhysicianFeeSched/PFS-Federal-Regulation-Notices-Items/CMS-1715-F

4. Basu S, Phillips RS, Bitton A, Song Z, Landon BE. Medicare Chronic Care Management Payments and Financial Returns to Primary Care Practices: A Modeling Study. Ann Intern Med. 2015;163(8):580-8. Available from: https://annals.org/acp/content_public/journal/aim/934607/ 0000605-201510200-00004.pdf. [Internet]. [cited 2019 Dec 2]

5. Medicare Provider Utilization and Payment Data: Physician and Other Supplier PUF CY2017 | Data.CMS.gov [Internet]. 2019 [cited 2019 Dec 8]. Available from: https://data.cms.gov/Medicare-Physician-Supplier/Medicare-Provider-Utilization-and-Payment-Data-Phy/fs4p-t5eq

6. Tai-Seale M, Olson CW, Li J, Chan AS, Morikawa C, Durbin M, et al. Electronic Health Record Logs Indicate That Physicians Split Time Evenly Between Seeing Patients And Desktop Medicine. Health Aff. 2017;36(4):655-62. https://doi.org/10.1377/hlthaff.2016.0811. [Internet] 